\title{
A second wave of COVID-19 infections should not lead to a new disruption of services in nuclear cardiology
}

On June 30, 2020, the Non-Communicable Diseases (NCDs) Department of the World Health Organization (WHO) published the results of a survey from 163 Ministries of Health, regarding the service delivery for NCDs during the coronavirus disease 19 (COVID-19) pandemic. ${ }^{1}$ A significant disruption of the services for cardiovascular disorders was reported, a finding that should be addressed properly, taking into consideration that these patients are at higher risk of increased COVID-19-related complications.

After the gradual relaxation of lockdown measures in Greece, we have managed to provide nuclear cardiology services at patient flow levels comparable to that of the pre-COVID-19 era. However, the indication of performing the examination is carefully evaluated, since referred patients are often high risk for adverse outcomes due to the COVID-19 infection. A new route to our Department was determined for non-hospitalized patients, avoiding potentially positive COVID-19 areas in the hospital. The intervals between patients' arrivals at the Department, and between consequent studies, have been extended resulting in the lengthening of the working day. Nuclear cardiology examinations are scheduled on specific days per week, and short-duration protocols are preferred (without compromising the quality of the studies). As the stress component in myocardial perfusion imaging studies, we favor the use of treadmill testing in patients able to exercise adequately, without any contraindications to dynamic stress. $^{2}$ On arrival and throughout their stay at the
Department, patients are instructed to follow the protective measures against COVID-19 spread, such as the use of a hand sanitizer on arrival and before leaving the Department, wearing masks, gloves and disposable gowns, and be seated in appropriate distance at the "hotroom". 3 Patients' body temperature is recorded on arrival. All staff members are aware of the importance of the early identification of suspect COVID-19 cases, and the required personal protective equipment (PPE) is constantly used by the staff. ${ }^{3}$ Surfaces, equipment and accessories are cleaned and/or disinfected regularly, based on current recommendations. Finally, the use of newer technologies has been encouraged for the communication with the patients, the referring physicians, and among staff members

In general, we have tried to standardize these procedures under the circumstances set by the COVID-19 pandemic. Later in the year, a second wave of COVID19 infections is quite possible. However, we believe that nuclear cardiology departments should be prepared to provide high-quality studies, taking all the appropriate measures for the prevention of viral spread. Proper adjustments based on the epidemic status are required, but a second disruption of nuclear cardiology services should be avoided.

G. Angelidis, MD, PhD, ${ }^{a}$ V. Valotassiou, $M D, P h D,{ }^{a}$ D. Psimadas, PhD, ${ }^{a}$ P. Georgoulias, $M D, P h D^{a}$

${ }^{a}$ Nuclear Medicine Laboratory, University of Thessaly, Larissa, Greece angelidis@protonmail.ch 


\section{Disclosures}

The authors George Angelidis, Varvara Valotassiou, Dimitrios Psimadas, and Panagiotis Georgoulias have nothing to declare.

\section{References}

1. Rapid assessment of service delivery for noncommunicable diseases (NCDs) during the COVID-19 pandemic. WHO NCD Department. file:///C:/Users/user/Downloads/for-web-rapid-assessment-30-june2020-(cleared).pdf.

2. Angelidis G, Valotassiou V, Psimadas D, Georgoulias P. Exercise stress testing: Time to restart in nuclear cardiology? J Nucl Cardiol. 2020. https://doi.org/10.1007/s12350-020-02234-w.
3. Huang HL, Gnanasegaran G, Paez D, et al. Nuclear medicine services after COVID-19: gearing up back to normality. Eur J Nucl Med Mol Imaging. 2020;47:2048-53.

doi:10.1007/s12350-020-02305-y

Publisher's Note Springer Nature remains neutral with regard to jurisdictional claims in published maps and institutional affiliations. 